\title{
Haplofungins, novel inositol phosphorylceramide synthase inhibitors, from Lauriomyces bellulus SANK 26899 I. Taxonomy, fermentation, isolation and biological activities
}

\author{
Takashi Ohnuki ${ }^{1}$, Tatsuya Yano $^{2}$, Yasunori Ono ${ }^{1}$, Shiho Kozuma $^{3}$, Toshihiro Suzuki ${ }^{4}$, Yasumasa Ogawa ${ }^{4}$ \\ and Toshio Takatsu ${ }^{1}$
}

\begin{abstract}
In the course of screening for antifungal agents, we have discovered eight novel compounds, haplofungin A, B, C, D, E, F, G and $\mathrm{H}$, from a culture broth of the fungus strain Lauriomyces bellulus SANK 26899. Haplofungins are composed of an arabinonic acid moiety linked through an ester to a modified long alkyl chain and show potent inhibitory activities against fungal inositol phosphorylceramide (IPC) synthase. Haplofungin A inhibited the activity of IPC synthase from Saccharomyces cerevisiae with an $\mathrm{IC}_{50}$ value of $0.0015 \mu \mathrm{g} \mathrm{ml}^{-1}$. This inhibitor also suppressed the growth of Candida glabrata at the MIC value of $0.5 \mu \mathrm{g} \mathrm{ml}^{-1}$. The Journal of Antibiotics (2009) 62, 545-549; doi:10.1038/ja.2009.72; published online 31 July 2009
\end{abstract}

Keywords: antifungal; haplofungin; inhibitor; inositol phosphorylceramide synthase; sphingolipid

\section{INTRODUCTION}

Opportunistic invasive fungal infections are a major cause of morbidity and mortality in cancer and immunocompromised patients. The frequency of such fungal infections is on the rise year after year. ${ }^{1}$ Moreover, the increase in aspergillosis and azole -resistant candidiasis makes chemotherapy difficult. ${ }^{2}$ Therefore, the discovery of antifungal agents having new mechanisms of action is anticipated.

Glycosphingolipid (GSL) is composed of a hydrophilic sugar chain and a ceramide that consists of hydrophobic sphingosine and fatty acid. In the animal kingdom, GSL is known to take part in a variety of life phenomena, such as cell differentiation and proliferation, organ formation, programmed cell death, etc. ${ }^{3}$ Similar to plants, the core structure of GSL in yeast and filamentous fungi consists of inositolphosphoceramide (IPC) in which phosphatidylinositol is linked to phytoceramide, and is quite different from that of mammalian GSL. ${ }^{4}$ It is therefore suggested that IPC synthase, an enzyme that synthesizes fungal-specific IPC from phytoceramide and phosphatidylinositol, is a novel target for antifungal drugs. ${ }^{5}$ Aureobasidin $\mathrm{A},{ }^{6}$ rustmicin ${ }^{7,8}$ and khafrefungin ${ }^{9}$ have been reported to inhibit IPC synthase, and also to suppress the growth of clinically important fungi such as Saccharomyces cerevisiae, Candida albicans and Cryptococcus neoformans. Recently, pleofungins were also reported by our group to be IPC synthase inhibitors. It is noteworthy that they suppressed the growth of Aspergillus fumigatus at the MIC value of $0.5 \mu \mathrm{g} \mathrm{ml}^{-1}$, in addition to the pathogenic fungi described above. ${ }^{10}$ From these results, the screening of IPC synthase inhibitors was suggested to be one of the most efficient ways to discover a novel broad-spectrum fungicide that has potent antifungal activities.

During the course of our continuous screening, novel fungicides named haplofungin A, B, C, D, E, F, G and H (Figure 1) were discovered from a culture broth of the fungus Lauriomyces bellulus SANK 26899. (These compounds showed potent inhibitory activities against the IPC synthase from S. cerevisiae.) In this paper, we report the taxonomy and fermentation of the producing fungus, as well as the isolation and biological properties of haplofungins. The structure determination and stereochemistry of haplofungins will be reported in succeeding papers.

\section{RESULTS}

Taxonomy of the producing organism

The producing microorganism, Lauriomyces bellulus Crous \& MJ Wingf. ${ }^{11}$ SANK 26899, was isolated from a soil sample collected in Ohota-shi, Shimane Prefecture, Japan.

Colonies on modified Weitzman and Silva-Hutner ${ }^{11}$ agar attained 21-22 $\mathrm{mm}$ in diameter in 7 days at $23^{\circ} \mathrm{C}$, plane (Figure 2). They were composed of a thin mycelia layer, white, sparse with sporulation toward the center and entire margins. Exudates and sclerotia were not observed. The mycelia were immersed and superficial, septate,

${ }^{1}$ Exploratory Research Laboratories I, Daiichi Sankyo Co. Ltd., 1-2-58 Hiromachi, Shinagawa-ku, Tokyo, Japan; ${ }^{2 B i o l o g i c a l ~ R e s e a r c h ~ L a b o r a t o r i e s ~ I I, ~ D a i i c h i ~ S a n k y o ~ C o . ~ L t d ., ~}$ 1-2-58 Hiromachi, Shinagawa-ku, Tokyo, Japan; ${ }^{3}$ Exploratory Research Laboratories II, Daiichi Sankyo Co. Ltd., 1-16-13, Kitakasai, Edogawa-ku, Tokyo, Japan and ${ }^{4}$ Process Development Laboratories, Daiichi Sankyo Co. Ltd., 389-4 Shimokawa Otsurugi, Izumimachi, Iwaki-shi, Fukushima, Japan

Correspondence: T Ohnuki, Exploratory Research Laboratories I, Daiichi Sankyo Co. Ltd., 1-2-58 Hiromachi, Shinagawa-ku, Tokyo 140-8710, Japan.

E-mail: ohnuki.takashi.eh@daiichisankyo.co.jp

Received 25 April 2009; revised 8 June 2009; accepted 18 June 2009; published online 31 July 2009 


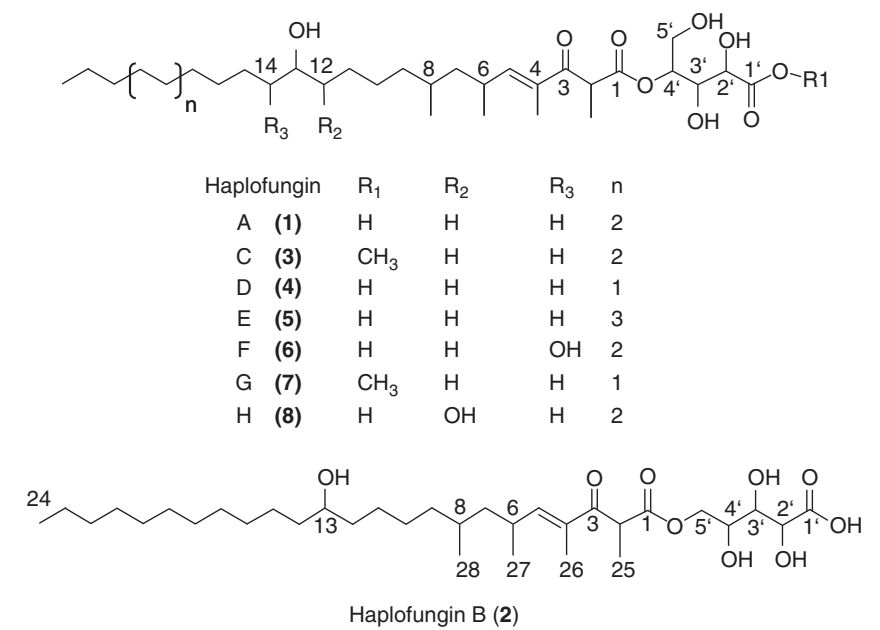

Figure 1 Structures of haplofungins.

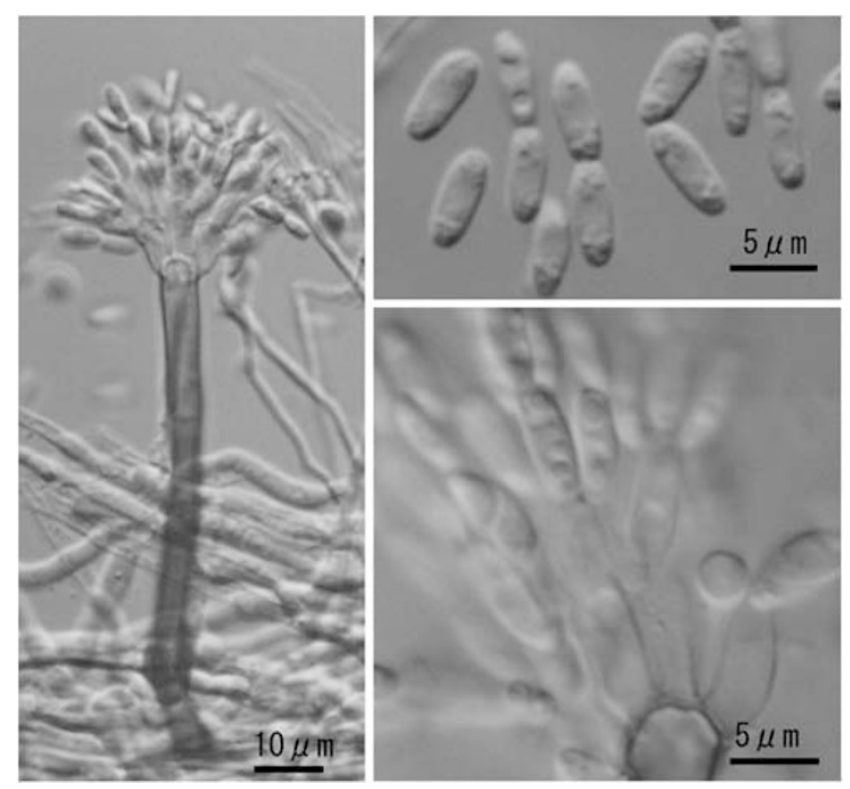

Figure 2 Microscopic characterisitics of Lauriomyces bellulus SANK 26899.

branched, smooth and hyaline, becoming brown near the conidiophores or setae. Setae were $440-650 \mu \mathrm{m}$ long, $4-6 \mu \mathrm{m}$ wide at the base, simple, solitary, erect, straight or curved, smooth, septate, dark brown and thick-walled, becoming tapered and light brown toward the apex. The conidiophores were 45-105 $\mu \mathrm{m}$ long, 4-6 $\mu \mathrm{m}$ wide, macronematous, mononematous, simple, solitary, erect, straight or curved, smooth, septate, dark brown and thick-walled at the base, becoming thin-walled and light brown toward the apex. The sporogenous apparatus was complex, consisting of a series of branches or conidiogenous cells arising from the stipe apex. The primary branches were 5-9 $\mu \mathrm{m}$ long, 3.5-5 $\mu \mathrm{m}$ wide, thin-walled, smooth and hyaline. The subsequent branches were hyaline and produced branches or conidia. The ramoconidia were $6-9 \times 2-3 \mu \mathrm{m}$, holoblastic, catenate, in acropetal branches or unbranched chains, aseptate, smooth, thinwalled, cylindrical to ellipsoidal, rarely curved, with flattened budding scars at both ends and hyaline.

In our previous patent application document, ${ }^{12}$ we reported that the producing strain had been identified as the fungus Haplographium

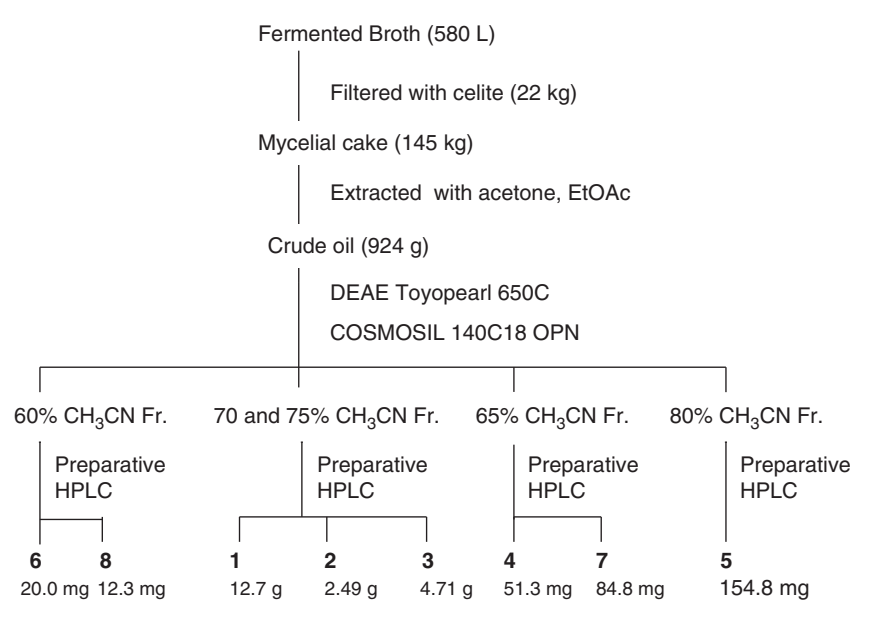

Figure 3 Isolation procedure for haplofungins.

heliocephalum V Rao and de Hoog SANK 26899, according to the description by Zucconi and Pagano ${ }^{13}$ and Rao and de Hoog, ${ }^{14}$ but $H$. heliocephalum was treated as a synonym of $L$. heliocephalus (Rao and de Hoog) Castañeda and Kendrick. ${ }^{11}$ In the course of establishing the genus Lauriomyces RF Castañeda, Castañeda and Kendrick ${ }^{11}$ recognized four species of Lauriomyces, with one of them being reported as L. heliocephalus. Subsequently, Crous and Wingfield added L. bellulus to the genus Lauriomyces, which resembles L. heliocephalus. ${ }^{15}$ These two species have similar-sized conidia, but can easily be distinguished from each other by the presence or absence of setae. This producing strain does not have setae and therefore SANK 26899 was identified as Lauriomyces bellulus Crous and MJ Wingf. ${ }^{15}$ It has been deposited with the International Patent Organism Depositary, National Institute of Advanced Industrial Science and Technology, Japan, under accession No. FERM BP-08506.

\section{Fermentation}

A seed medium $(500 \mathrm{ml})$ consisting of $3 \%$ glycerin, $3 \%$ glucose, $2 \%$ soluble starch, $1 \%$ soybean meal, $0.25 \%$ gelatin, $0.25 \%$ yeast extract, $0.25 \% \mathrm{NH}_{4} \mathrm{NO}_{3}$ and $0.01 \% \mathrm{CB}-442(\mathrm{pH} 7.0$ ) was poured into each of two 2-l Erlenmeyer flasks and sterilized at $121^{\circ} \mathrm{C}$ for $30 \mathrm{~min}$. A loopful of the slant culture of the strain, SANK 26899, was inoculated to each medium and cultured on a rotary shaker $\left(210\right.$ r.p.m.) at $23^{\circ} \mathrm{C}$ for 6 days. A second seed medium (30l) with the same contents was poured into each of two $60-1$ jar fermentors and sterilized at $121^{\circ} \mathrm{C}$ for $30 \mathrm{~min}$. Then, the first seed culture (5\%) was transferred to the fermentors and cultivation was carried out at $23^{\circ} \mathrm{C}$ for 2 days. A production medium (3001), consisting of $8 \%$ glycerin, $0.25 \%$ gelatin, $1 \%$ soybean meal, $0.25 \%$ yeast extract, $0.25 \% \mathrm{NH}_{2} \mathrm{PO}_{4}, 0.1 \%\left(\mathrm{NH}_{4}\right)_{2} \mathrm{SO}_{4}$ and $0.01 \%$ CB-442 (pH 7.0) in each of two 600-1 tank fermentors, was sterilized at $121{ }^{\circ} \mathrm{C}$ for $30 \mathrm{~min}$. The second seed culture (5\%) was transferred to the production medium and fermentation was carried out at $23^{\circ} \mathrm{C}$ for 7 days.

\section{Isolation and purification of haplofungins}

The isolation and purification procedures for haplofungins are shown in Figure 3. The culture broth (5801) was filtered with Celite 545 $(22 \mathrm{~kg})$ as a coadjuvant to separate the supernatant and mycelial cake. The mycelial cake $(145 \mathrm{~kg})$ was soaked in acetone (240l) and filtered. Ethyl acetate (240l) was added to the extract and subsequently partitioned by the addition of $0.1 \%$ phosphoric acid (1201). The organic layer was washed with a saturated $\mathrm{NaCl}$ solution, dried over 
anhydrous $\mathrm{Na}_{2} \mathrm{SO}_{4}$ and concentrated in vacuo to give brown oil (924 g). The oily substance was dissolved in $100 \mathrm{ml}$ of hexane-ethyl acetate-methanol $(1: 1: 1)$ and applied to a DEAE Toyopearl $650 \mathrm{C}$ column $\left(\mathrm{AcO}^{-}\right.$type, 61, Tosoh, Tokyo, Japan) equilibrated with ethyl acetate. Further, the column was washed with hexane-ethyl acetate (1:1, 12l), ethyl acetate (12l) and methanol (12l) and the active substance was eluted with methanol-acetic acid (9:1, 12l). Then, the eluate was concentrated in vacuo to dryness and the residue $(338 \mathrm{~g})$ was dissolved in $50 \%$ aqueous acetonitrile containing $0.1 \%$ phosphoric acid. This solution was applied to a COSMOSIL 140C18 OPN column (81, Nacalai Tesque, Kyoto, Japan). The chromatography was performed stepwise with $60,65,70,75$ and $80 \%$ aqueous acetonitrile containing $0.1 \%$ phosphoric acid. The eluted fractions were further purified as follows.

Isolation of haplofungin $A$ (1), B (2) and C (3). Seventy and seventyfive percent acetonitrile fractions were combined and evaporated in vacuo to remove the acetonitrile and extracted with ethyl acetate $(500 \mathrm{ml})$. The organic layer was dried over anhydrous $\mathrm{Na}_{2} \mathrm{SO}_{4}$ and concentrated in vacuo to dryness to give a residue. The residue was dissolved in $77 \%$ aqueous acetonitrile containing $0.1 \%$ phosphoric acid, applied to an ODS HPLC column (YMC-pack ODS-AM, $100 \times 500 \mathrm{~mm}$, YMC, Kyoto, Japan) and chromatographed with $80 \%$ aqueous acetonitrile containing $0.1 \%$ phosphoric acid to obtain three active fractions. Each of the active fractions was evaporated in vacuo to dryness to remove the acetonitrile and was extracted with ethyl acetate. The ethyl acetate layer was dried over anhydrous $\mathrm{Na}_{2} \mathrm{SO}_{4}$ and concentrated in vacuo to give haplofungin A $(1,12.7 \mathrm{~g}), \mathrm{B}(2,2.49 \mathrm{~g})$ and $\mathrm{C}(3,4.71 \mathrm{~g})$.

Isolation of haplofungin D (4) and G (7). A 65\% acetonitrile fraction was extracted with ethyl acetate. The organic layer was dried over anhydrous $\mathrm{Na}_{2} \mathrm{SO}_{4}$ and concentrated in vacuo to dryness. The residue was applied to an ODS HPLC column (Develosil ODS HG-5, $20 \times 150 \mathrm{~mm}$, Nomura Chemical, Aichi, Japan) and chromatographed with $77 \%$ aqueous acetonitrile containing $0.1 \%$ formic acid. The active fractions were concentrated in vacuo and lyophilized to give haplofungin $\mathrm{D}(4,51.3 \mathrm{mg})$ and $\mathrm{G}(7,84.8 \mathrm{mg})$.

Isolation of haplofungin E (5). An $80 \%$ acetonitrile fraction was extracted with ethyl acetate. The organic layer was dried over anhydrous $\mathrm{Na}_{2} \mathrm{SO}_{4}$ and concentrated to dryness. The residue was applied to an ODS HPLC column (Develosil ODS HG-5, 20×150 mm) and chromatographed with $85 \%$ aqueous acetonitrile containing $0.1 \%$ phosphoric acid. The active fractions were concentrated in vacuo to remove the acetonitrile and extracted with ethyl acetate. The ethyl acetate layer was concentrated in vacuo to dryness to give haplofungin E $(5,154.8 \mathrm{mg})$.

Isolation of haplofungin $F(6)$ and $H(8)$. A 60\% acetonitrile fraction was evaporated in vacuo to remove the acetonitrile and extracted with ethyl acetate. The organic layer was concentrated in vacuo and the residue was applied to an ODS HPLC column (YMC-pack ODS-AM, $100 \times 500 \mathrm{~mm}$ ) and chromatographed with $65 \%$ aqueous acetonitrile containing $0.1 \%$ phosphoric acid. The active fraction was evaporated in vacuo to remove the acetonitrile and extracted with ethyl acetate. The active fraction was re-chromatographed with an ODS HPLC column ( $\mu$ BONDASPHERE, $50 \times 100 \mathrm{~mm}$, Waters, Milford, MA, USA) and eluted with $60 \%$ aqueous acetonitrile containing $0.1 \%$ phosphoric acid. The active fraction was evaporated in vacuo to remove the acetonitrile and extracted with ethyl acetate. The organic layer was dried over anhydrous $\mathrm{Na}_{2} \mathrm{SO}_{4}$ and concentrated to dryness.
Table 1 Inhibitory activity of haplofungins against IPC synthase prepared from $S$. cerevisiae and $A$. fumigatus

\begin{tabular}{lll}
\hline & \multicolumn{2}{c}{$1 C_{50}(\mu \mathrm{g} / \mathrm{m} /)$} \\
\cline { 2 - 3 } Compounds & S. cerevisiae & A. fumigatus \\
\hline $\mathbf{1}$ & 0.0015 & 0.25 \\
$\mathbf{2}$ & 0.025 & 0.8 \\
$\mathbf{3}$ & 0.010 & 0.5 \\
$\mathbf{4}$ & 0.054 & 0.6 \\
$\mathbf{5}$ & 0.0015 & 0.24 \\
$\mathbf{6}$ & 0.053 & 1.3 \\
$\mathbf{7}$ & 0.059 & 2 \\
$\mathbf{8}$ & 0.035 & 0.8 \\
Pleofungin A & 0.04 & 0.0095 \\
Khafrefungin & 0.065 & 0.5 \\
\hline
\end{tabular}

The active substances were finally separated by ODS HPLC (Develosil ODS HG-5, $20 \times 150 \mathrm{~mm}$ ) with $65 \%$ aqueous acetonitrile containing $0.1 \%$ formic acid. Each of the active fractions was concentrated in vacuo and lyophilized to give haplofungin $\mathrm{F}(6,20.0 \mathrm{mg})$ and $\mathrm{H}(\mathbf{8}, 12.3 \mathrm{mg})$.

\section{Biological properties of haplofungins}

The inhibitory activities of haplofungins and other inhibitors against IPC synthase prepared from S. cerevisiae and A. fumigatus are summarized in Table 1. All of the haplofungins inhibited IPC synthases from these fungi. Although $\mathbf{1}$ and $\mathbf{5}$ were the most potent compounds with an approximate $\mathrm{IC}_{50}$ value of $1.5 \mathrm{ng} \mathrm{ml}^{-1}$ against the IPC synthase from $S$. cerevisiae, the other related compounds had less activity. Compound 3, the methyl ester of 1 , was 6 -fold less potent than 1 and the results indicated that $\mathrm{IC}_{50}$ was affected by the presence of methyl ester in the aldonate moiety. A similar pattern was observed between 5 and 7. Compound 7, the methyl ester derivative of $\mathbf{5}$, showed 40-fold less potent inhibitory activity against the enzyme from S. cerevisiae. The dihydroxyl analogs, $\mathbf{6}$ and $\mathbf{8}$, had less enzyme inhibitory activity than $\mathbf{1}$ and the others. Thus, the existence of a hydroxyl group, except at C-13 in the alkyl chain, also reduced the $\mathrm{IC}_{50}$ value. The alkyl chain length of haplofungins is a very important factor for inhibitory activity. The $\mathrm{IC}_{50}$ values of $\mathbf{1}, 4$ and 5 indicate that the IPC synthase inhibitory activity of haplofungins depends on the alkyl chain length. Compound 2, which has an aldonate moiety attached to the C-1 ester carbonyl groups at C-5', had less inhibitory activity than 1. Therefore, the results indicate that an aldonate moiety attached to C-1 at C-4' is essential for eliciting IPC synthase inhibitory activity.

The in vitro antifungal activities of haplofungins and the other compounds are summarized in Table 2. In spite of their potent IPC synthase activities, haplofungins did not display any antifungal activity, except for C. glabrata. It is suggested that poor membrane permeability or accessibility to the target enzyme of the compounds may affect antifungal activity.

\section{DISCUSSION}

We isolated novel compounds, haplofungins, and found that these compounds are potent IPC synthase inhibitors. Haplofungins were produced by the fungal strain, SANK 26899, which was identified as Lauriomyces bellulus.

As shown in Tables 1 and 2, 1 inhibited IPC synthase activity from S. cerevisiae with an $\mathrm{IC}_{50}$ value of $1.5 \mathrm{ng} \mathrm{ml}^{-1}$ and also suppressed the growth of C. glabrata at the MIC value of $0.5 \mu \mathrm{g} \mathrm{ml}^{-1}$. 
Table 2 In vitro antifungal activities of haplofungins and other IPC synthase inhibitors

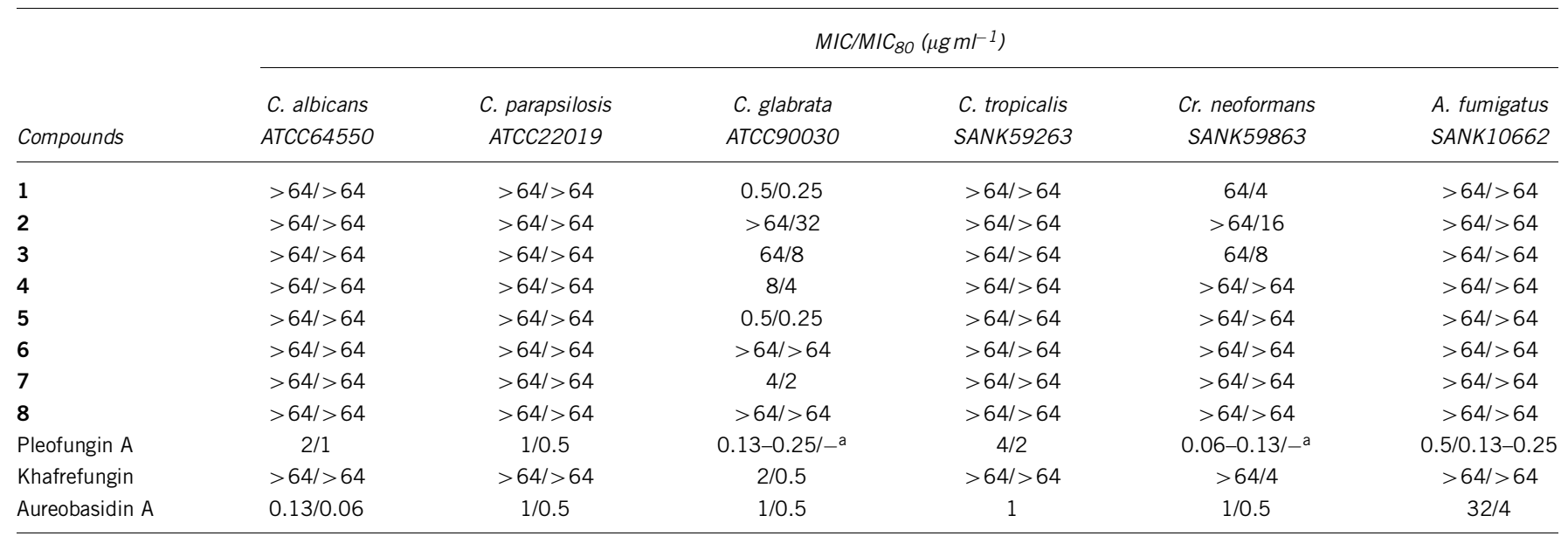

aNot tested.

When the inhibitory activities of these compounds were tested against an enzyme from $A$. fumigatus, they showed 10- to 100 -fold less potent activities than in the case of $S$. cerevisiae. In particular, the IPC synthase from $A$. fumigatus was significantly less sensitive to $\mathbf{1}$ and $\mathbf{5}$, with an approximate $\mathrm{IC}_{50}$ of $0.25 \mu \mathrm{g} \mathrm{ml}^{-1}$, which is 170 -fold less potency.

Khafrefungin was reported as an IPC synthase inhibitor isolated from the culture broth of the strain MF6020 by the Merck Group (Whitehouse Station, NJ, USA). ${ }^{9}$ The structure is very similar to haplofungins in terms of the structural components of aldonic acid and a modified alkyl chain. The absolute configuration was determined by total synthesis ${ }^{16}$ and the aldonic acid moiety was determined to be an arabinonic acid, the same as haplofungins. As shown in Table 1, Khafrefungin also inhibited IPC synthases from C. albicans and A. fumigatus; however, the activities were 40 -fold less active than 1. Therefore, it is supposed that the structure of a modified C24 alkyl chain moiety in $\mathbf{1}$ is essential for potent inhibitory activity against the IPC synthase of $S$. cerevisiae. Pleofungin $\mathrm{A}^{10,17}$ is a cyclic depsipeptide isolated as an IPC synthase inhibitor. Although this compound showed 26-fold more potent inhibitory activity than $\mathbf{1}$ against the IPC synthase of $A$. fumigatus, it had lower inhibitory activity than $\mathbf{1}$ against the IPC synthase of $S$. cerevisiae. ${ }^{10}$ It is interesting to note that the inhibitory activities of the two distinct types of inhibitors were greatly different. It has been reported that the homology of IPC synthase genes between filamentous fungi and yeast is low. ${ }^{18,19}$ These results indicated that the difference in the affinity to the enzyme of these two types of inhibitors may be affected by the antifungal spectrum.

From the viewpoint of the structure-activity relationship, the inhibitory activities of these compounds against IPC synthase indicated that the free arabinonic acid moiety and modified long alkyl chain with one hydroxyl group at C-13 were suggested to be essential for eliciting IPC synthase inhibitory activity. It might be possible that the core structure of haplofungins mimics the structure of phytoceramide, which is the substrate of IPC synthase. If the antifungal activity is improved by structure modification, there is a possibility that haplofungin-derived compounds may be candidates for more safe and effective antifungal agents.

\section{MATERIALS AND METHODS}

\section{General experimental procedures}

The strain, SANK 26899, was isolated from a soil sample collected in Ohota-Shi, Shimane Prefecture. This strain was used for the production of haplofungins. For determination of the amounts of haplofungins in the culture broth, the samples were analyzed on an 1100 HPLC system (Agilent Technologies, Santa Clara, CA, USA) using a reverse phase column (Cadenza CD-C18, $4.6 \times 75 \mathrm{~mm}$, Imtakt, Kyoto, Japan); flow rate, $0.6 \mathrm{ml} \mathrm{min}^{-1}$; mobile phase, a 15 -min linear gradient from $65 \%$ acetonitrile containing $0.1 \%$ phosphoric acid to $95 \%$ acetonitrile containing $0.1 \%$ phosphoric acid; detection, UV at $230 \mathrm{~nm}$.

\section{Compounds}

Pleofungin A, aureobasidin A and khafrefungin were purified from the original strains of Daiichi-Sankyo (Toyko, Japan). Phosphatidyl inositol was purchased from Sigma-Aldrich (St Louis, MO, USA) and BODIPY-FL-C5-ceramide was purchased from Molecular Probes (Carlsbad, CA, USA).

\section{IPC synthase assay}

The method for measuring the inhibitory activity against IPC synthases was previously described. ${ }^{10}$ We used Saccharomyces cerevisiae and Aspergillus fumigatus as the sources of IPC synthase. Crude IPC synthase of A. fumigatus and S. cerevisiae was prepared from stationary growing fungal cells cultured in yeast extract-peptone-dextrose medium. To measure the IPC synthase activities in vitro, crude enzyme was mixed with BODIPY-FL-C5-ceramide and phosphatidyl inositol. After incubation of the reaction mixtures, BODIPYlabeled IPC was extracted by the addition of solvent mixture (MeOH:diethylether, 41:91) and their fluorescent values were measured (Ex/Em, 505/513).

\section{Antifungal activities}

The minimum inhibitory concentrations were evaluated by the microtiter broth dilution method in RPMI-1640 medium. ${ }^{20}$ For the determination of the minimum inhibitory concentrations against Candida species and Cryptococcus neoformans, cells were diluted into $5.0 \times 10^{3} \mathrm{CFU} / \mathrm{ml}$ in RPMI medium. The cell suspensions $(100 \mu \mathrm{l})$ were transferred into 96 -well microtiter plates. Then, $100 \mu \mathrm{l}$ of diluted samples with RPMI medium was added to the cell suspensions. After mixing the samples and cell suspensions of Candida and Cryptococcus, the plates were incubated at $37^{\circ} \mathrm{C}$ for 24 and $48 \mathrm{~h}$, respectively. For the minimum inhibitory concentrations against the Aspergillus species, cell suspensions $(90 \mu \mathrm{l})$ were distributed into 96-well microtiter plates and $100 \mu \mathrm{l}$ of diluted samples and $10 \mu \mathrm{l}$ of Alamar Blue were further added. Then, the plates were incubated at $26^{\circ} \mathrm{C}$ for $72 \mathrm{~h}$ and the growth conditions were determined by measuring $\mathrm{OD}_{595}$.

1 Müller, J. Epidemiology of deep-seated, domestic mycoses. Mycoses 37, 1-7 (1994).

2 Rex, J. H. \& Pfaller, M. A. Resistance of Candida species to fluconazole. Antimicrob. Agents Chemother. 39, 1-8 (1995). 
3 Dickson, R. C. \& Lester, R. L. Sphingolipid functions in Saccharomyces cerevisiae. Biochim. Biophys. Acta. 1583, 13-25 (2002).

4 Dickson, R. C. Sphingolipid functions in Saccharomyces cerevisiae: comparison to mammals. Ann. Rev. Biochem. 67, 27-48 (1998).

5 Sugimoto, Y. \& Yamada, K. IPC synthase as a useful target for antifungal drugs. Curr. Drug. Targets. Infect. Disord. 4, 311-322 (2004).

6 Takesako, K. et al. Biological properties of aureobasidin A, a cyclic depsipeptide antifungal antibiotic. J. Antibiot. 46, 1414-1420 (1993).

7 Takatsu, T. et al. Rustmicin, a new macrolide antibiotic active against wheat stem rust fungus. J. Antibiot. 38, 1806-1809 (1985).

8 Mandala, S. M. et al. Rustmicin, a potent antifungal agent, inhibits sphingolipid synthesis at inositol phosphoceramide synthase. J. Biol. Chem. 273, 14942-14949 (1998).

9 Mandala, S. M. et al. Khafrefungin, a novel inhibitor of sphingolipid synthesis. J. Biol. Chem. 272, 32709-32714 (1997).

10 Yano, T. et al. Pleofungins, novel inositol phosphorylceramide synthase inhibitors, from Phoma sp. SANK 13899. I. Taxonomy, fermentation, isolation, and biological activities. J. Antibiot. 60, 136-142 (2007).

11 Castañeda, R. F. \& Kendrick, B. Conidial fungi from Cuba: 1. Univ Waterloo Biol Ser 32, 21 (1990).

12 Ohnuki, T., Takatsu, T., Yano, T. \& Ono, Y. (Sankyo, Co. LTD.), Novel anti fungal compound of F-15949. JP252834, September 10 (2003).
13 Zucconi, L. \& Pagano, S. Concerning the generic limits in Haplographium. Mycotaxon 66, 11-18 (1993).

14 Rao, V. \& de Hoog, G. S. New or critical Hyphomycetes from India. Stud. Mycol. 28, 1-84 (1986).

15 Crous, P. W. \& Wingfield, M. J. Sporendocladia fumosa and Lauriomyces bellulus sp. nov. from Castanea cupules in Switzerland. Sydowia 46, 193-203 (1994).

16 Wakabayashi, T., Mori, K. \& Kobayashi, S. Total synthesis and structure elucidation of khafrefungin. J. Am. Chem. Soc. 123, 1372 (2001).

17 Aoyagi, A., Yano, T., Kozuma, S. \& Takatsu, T. Pleofungins, novel inositol phosphorylceramide synthase inhibitors, from Phoma $s p$. SANK 13899. II. Structural elucidation. J. Antibiot. 60, 143-152 (2007).

18 Heidler, S. A. \& Radding, J. A. Inositol phosphoryl transferase from pathogenic fungi. Biochem. Biophys. Acta 1500, 147-152 (2000).

19 Zhong, W., Jeffries, M. W. \& Georgopapadakou, N. H. Inhibition of inositol phosphorylceramide synthase by aureobasidin A in Candida and Aspergillus Species. Antimicrob. Agents and Chemother. 44, 651-653 (2000).

20 Barchiesi, F., Colombo, A. L., McGough, D. A. \& Rinaldi, M. G. Comparative study of broth macrodilution and microdilution techniques for in vitro antifungal susceptibility testing of yeasts by using the National Committee for Clinical Laboratory Standards' proposed standard. J. Clin. Microbiol. 32, 2494-2500 (1994). 